\title{
LE DÉVELOPPEMENT DE LA GONADE DES FREEMARTINS
}

\author{
A. JOST, B. VIGIER, J. PRÉPIN et J.-P. PERCHELLET \\ Laboratoire de Physiologie comparée, \\ Universitê Paris VI, \\ 9, quai Saint-Bernard, \\ 75005 Paris (France)
}

\section{RÉSUMÉ}

La différenciation des gonades a été étudiée chez les 82 fœtus freemartins prélevés entre 40 et 250 jours. Les portées multiples comportant des foetus des deux sexes ont été induites par les hormones gonadotropes ou obtenues aux abattoirs.

L'histoire de la gonade des fotus freemartins peut être divisée en quatre périodes.

Pendant la première semaine suivant la différenciation testiculaire des mâles (40-48 jours), la gonade des freemartins présomptifs demeure semblable à l'ébauche gonadique indifférenciée des femelles témoins.

Entre 48 et 60 jours, la croissance de la gonade des freemartins et la multiplication des cellules germinales sont arrêtées. Simultanément, la partie antérieure des canaux de Müller commence à régresser, mais il n'y a aucun signe de masculinisation.

De 60 à 90 jours, la gonade des freemartins reste très petite et les derniers îlots de cellules germinales périphériques disparaissent. De légers signes de stimulation androgénique peuvent être observés au niveau des vésicules séminales et parfois de l'épididyme.

Après go jours, au moment où commence la différenciation des follicules ovariens chez les femelles normales, la croissance de la gonade des freemartins reprend. Dans environ la moitié des cas, il s'y développe des formations ressemblant à des cordons séminifères plus ou moins bien différenciés, certains isolés dans le blastème gonadique, $d$ 'autres en relation avec le rete. Le volume du rete s'est alors considérablement accru. Dans quelques cas, des îlots de cellules interstitielles de type testiculaire sont reconnaissables. Chez les trois foetus dont les gonades étaient les plus transformées dans le sens testiculaire, le rete était le plus volumineux et le tractus génital le plus masculinisé ; la prostate, notamment, s'était développée.

Les gonades des freemartins des bovidés posent un intéressant problème : bien qu'appartenant à des animaux de sexe génétique féminin, elles montrent à la naissance diverses caractéristiques masculines, comme l'ont rapporté les premiers auteurs qui en ont fait 1'étude histologique (LILIIE, I9I7; CHAPIN, I9I7 ; WILLIER, I92I ; LILLIE, I923 ; BISSONNETTE, I924, I928). La structure de ces gonades est très variable. Les moins atteintes peuvent contenir des follicules ovariens pourvus d'ovocytes (ChaPIN, I9I7 ; RAJAKOSKI et HAFEz, I963), mais il s'agit là de cas exceptionnels. 
En général, ces gonades ont un volume très réduit, elles sont limitées en surface par une albuginée fibreuse, et, dans un stroma conjonctif irrégulier, elles contiennent souvent des structures identifiées par les auteurs à des tubes séminifères et des cellules interstitielles de type testiculaire. Les tubes séminifères n'ont qu'exceptionnellement été décrits chez des fœetus et, dans ce cas, chez des fœetus de plus de $20 \mathrm{~cm}$ (ChAPIN, I9I7 ; BissonNeTTE, I924; HAY, I950). On en a décrit juste à la naissance (OHNo et al., 1962) et bien entendu chez les freemartins plus âgés. Les cellules germinales présentes dans les tubes séminifères avant la naissance (CHAPIN, I9I7 ; HAY, I950), semblent disparaître ultérieurement (WILLIER, I92I ; SHORT et al., I969) et les tubes séminifères sont alors stériles. Des cellules interstitielles semblables à celles du testicule ont été décrites chez un freemartin de $25 \mathrm{~cm}(\mathrm{HAY}$, I950) et chez plusieurs freemartins après la naissance. Ce fait est à mettre en relation avec la sécrétion de testostérone, mise en évidence dans la veine gonadique d'un freemartin d'un an particulièrement masculinisé (SHORT et al., I969).

Jusqu'ici aucune étude systématique du développement des gonades des freemartins n'a été faite ; les modalités de l'organogenèse de l'ovaire normal sont ellesmêmes insuffisamment connues. Les premières interprétations de la gonade des freemartins ont été basées sur une théorie classique mais inexacte du développement de la gonade des Mammifères, selon laquelle des cordons sexuels poussent en profondeur, à partir de l'épithélium superficiel : une première génération de cordons (cordons sexuels primaires) donnerait les tubes séminifères du testicule, et des "cordons médullaires "dans l'ovaire ; le cortex ovarien définitif résulterait d'une deuxième poussée de cordons sexuels (dits cordons de Pflüger). En décrivant les gonades des freemartins ChaprN (I9I7), WILIIER (I92I), LILIIE (I9I7, I923) et BISSONNETTE (I924), ont insisté sur l'arrêt de développement des " cordons de Pflüger " et ont assimilé les tubes séminifères, quand ils sont présents, aux "cordons sexuels primaires" Cependant BissonNETTE (I928) a un doute et soulève la question suivante : " the question as to how much of the definitive seminiferous tubules of the freemartin testis comes from primary or medullary sex cords, and how much from secondary sex cord or cortical regions of the early freemartin ovary " (page 46).

Il y a longtemps que divers auteurs ont critiqué ou rejeté la théorie des cordons sexuels invaginés à partir de l'épithélium superficiel (cf. PRENANT, I889; Fischei, I930). En ce qui concerne le veau, il en est ainsi de VAN BEEK (I924), qui en cent pages assez difficiles à lire et peu illustrées, décrit l'organogenèse ovarienne du veau. Dans l'ébauche du fœtus de $3 \mathrm{rmm}$ (46 jours environ), il distingue : I) l'épithélium germinatif ; 2) en-dessous, une corticalis épaisse de I2 cellules environ ; 3) une zone lâche de transition ; 4) un noyau conjonctivo-épithélial irrégulier, ou medullaris. Dans la corticalis se formeront les nids à ovogonies ou cordons corticaux, au contact de l'épithélium, mais " die Corticalstränge im Rindenovarium rïhren nicht von einer besonderen Keimepithelproliferation her ".

Dans la medullaris se différencieront d'une part le rete ovarii, et d'autre part des cordons médullaires ("Markstränge ») visibles à partir du stade de $60 \mathrm{~mm}$; ces cordons deviennent plus nets ensuite, acquièrent parfois une lumière $(82 \mathrm{~mm})$ et entrent en contact avec les nids ovigères de la corticalis à partir de $90 \mathrm{~mm}$. Au stade de I04 mm (environ 70 jours), certaines cellules germinales ont atteint le stade synaptène et pachytène. L'auteur ne décrit guère la formation des premiers follicules. Gropp et OHNo (Ig66), utilisant une technique histochimique de mise en évidence 
de la phosphatase alcaline, insistent sur l'existence dans l'ébauche gonadique d'un " blastème central " dans lequel se forment les tubes séminifères du mâle, et qui chez la femelle formera des " cordons folliculaires " qui se mettront en contact avec les nids à ovocytes et fourniront les cellules folliculaires. Les auteurs rejettent également la participation de l'épithélium germinatif à la formation de cordons sexuels ou de cordons de Pflüger. OHNo (I969), après avoir étudié des fœtus freemartins de 235 à $270 \mathrm{~mm}$, explique la structure de la gonade des freemartins et sa stérilité, par la dégénérescence de la zone corticale externe et par la différenciation en tubes de la zone centrale.

Nous avons indiqué antérieurement que dans l'ovaire présomptif du veau normal ou dans la gonade des freemartins jusqu'au stade de 60 jours après l'insémination, il n'existe aucune structure comparable à des cordons sexuels primaires (JoST, I970 ; Jost, Vigier et PrépIN, I972). Nous en déduisions que lorsque des tubes séminifères se forment dans ces gonades, ils doivent le faire après le stade de 60 jours. C'est ce que nous avons observé, et nous rapportons ici un résumé d'ensemble de nos observations dont divers aspects ont été mentionnés ailleurs (Jost et al., I973).

Auparavant, il nous semble nécessaire de donner la définition des termes de testicule et d'ovaire, tels que nous les utiliserons. Le testicule est un organe constitué par : r) des tubes séminifères, qui chez le fotus sont des cordons pleins, constitués par des cellules de Sertoli fotales et des cellules germinales ; chez le veau, les premiers cordons séminifères se forment, à quelque distance sous l'épithélium superficiel, entre 39 et $4 \mathrm{I}$ jours. Plus tard, après 56 jours, ces cordons se mettent en relation avec les tubes du rete par des tubes droits ; 2) des cellules interstitielles, dont la différenciation suit celle des premiers cordons séminifères ; 3) une albuginée, qui devient très nette déjà entre 43 et 45 jours.

L'ovaire se différencie beaucoup plus tard que le testicule. Pendant une longue période durant laquelle les mâles ont déjà des testicules bien reconnaissables, les femelles ont encore des ébauches de gonades indifférenciées. Les auteurs (et nousmêmes dans le passé) appellent ces organes des ovaires, parce que ce ne sont pas des testicules et parce que normalement ils se développent en ovaires. Mais en fait ce ne sont que des ovaires présomptifs dont les freemartins montrent précisément qu'ils peuvent subir une différenciation qui n'est pas de type ovarien. Nous considérerons que la gonade indifférenciée est devenue un ovaire, seulement lorsqu'elle contiendra des follicules primordiaux séparés par un stroma, l'ensemble étant recouvert par un épithélium ovarien superficiel. C'est une règle semble-t-il générale chez les Mammifères, que les cellules germinales entrent en méiose avant d'être entourées par les cellules f $\supset$ lliculaires, donc avant que la structure ovarienne ne se réalise. Chez le veau, les premières figures de méiose ovocytaire apparaissent vers 75 jours (ERICKson, I 966 ) et les premiers follicules se constituent vers Ioo jours. La formation de follicules et l'organogenèse ovarienne se poursuivent pendant de nombreuses semaines.

\section{MATÉRIEL ET MÉTHODES}

Les foetus freemartins de notre première collection (âgés de 39 à 77 jours) ont été obtenus à la Station de Physiologie animale de 1'I. N. R. A., à Jouy-en-Josas, après induction hormonale de la superovulation selon la technique rapportée par Mauléon et al. (1970). Les fœetus plus âgés 
ont été collectés aux abattoirs grâce à l'obligeance des Laboratoires Sorga. Le nombre des fœetus étudiés est indiqué dans le tableau r. L'ensemble des techniques et les critères utilisés pour considérer un fœtus comme un freemartin en développement ont été discutés antérieurement (Jost et al., 1972).

Le matériel a été fixé par le liquide de Bouin et coupé en série à $7 \mu \mathrm{m}$ d'épaisseur. On a en général monté et étudié d'abord une coupe sur 33 ; ensuite on a prélevé, selon les niveaux, des coupes à intervalles plus rapprochés ou toutes les coupes intermédiaires. Les mesures de volume ont été faites selon la méthode de projection et de pesée des dessins. Le nombre des cellules germinales dans les gonades a été obtenu selon la méthode de CHALKLEY (I.943).

TABLEAU $\boldsymbol{I}$

Nombre de foetus étudiés

\begin{tabular}{l|c|c|c}
\hline Age (jours) & Freemartins & Femelles & Mâles \\
\hline $40-48$ & 14 & 6 & 18 \\
$49-60$ & 22 & 26 & 31 \\
$61-90$ & 15 & 16 & 11 \\
$91-250$ & 31 & 13 & 1 \\
& -62 & 61 & 61 \\
\hline
\end{tabular}

\section{RÉSULTATS}

Les observations seront rapportées en distinguant quatre classes d'âge qui tiennent compte de certains événements importants survenant chez les freemartins ou chez les témoins.

\section{0 à 48 jours}

Chez les mâles, la différenciation testiculaire progresse rapidement, alors que chez les femelles et les freemartins l'ébauche gonadique reste indifférenciée : un blastème cellulaire se distingue de plus en plus nettement dans le centre de la gonade, séparé par une zone plus lâche, d'une zone périphérique assez mince et limitée par l'épithélium superficiel. Le volume des gonades des freemartins est peut-être légèrement inférieur à celui des gonades témoins (fig. I) et le nombre de cellules germinales un peu plus faible (fig. 2), mais ceci demanderait à être vérifié sur d'autres animaux, parce que certains de nos freemartins jeunes, ainsi que leurs jumeaux mâles, étaient un peu retardés dans leur développement.

\section{9 à 60 jours}

Cette période est particulièrement importante dans l'histoire des mâles, puisqu'elle correspond à la masculinisation de l'ensemble de l'appareil génital et à la régression de la partie supérieure des canaux de Müller.

Chez les femelles, le fait le plus saillant est la rapide multiplication des cellules 


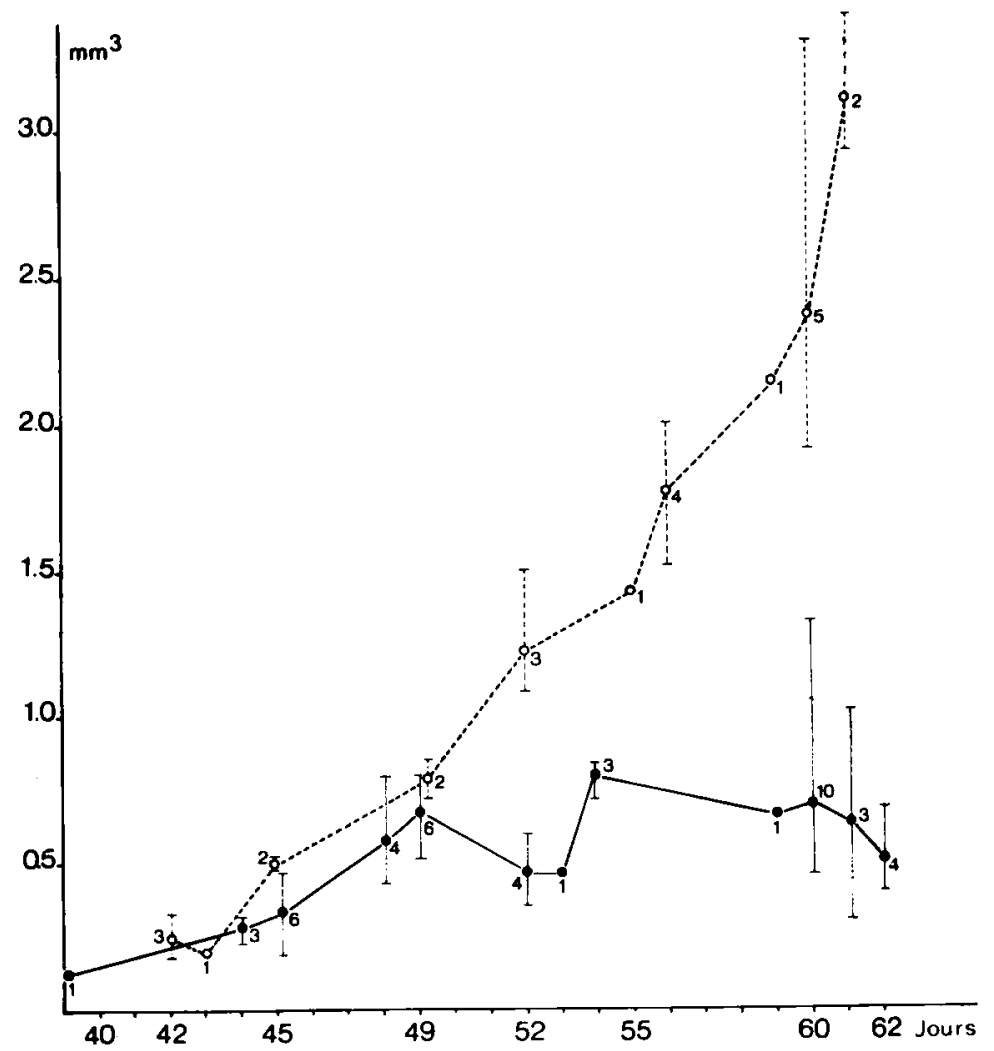

FIG. I. - Volume moyen des gonades gauches chez des freemartins et des femelles témoins de 39 à 62 jours (animaux cités dans Jost et al., 1972)

On a indiqué le nombre de cas étudiés pour chaque stade, ainsi que les valeurs extrêmes des volumes gonadiques.

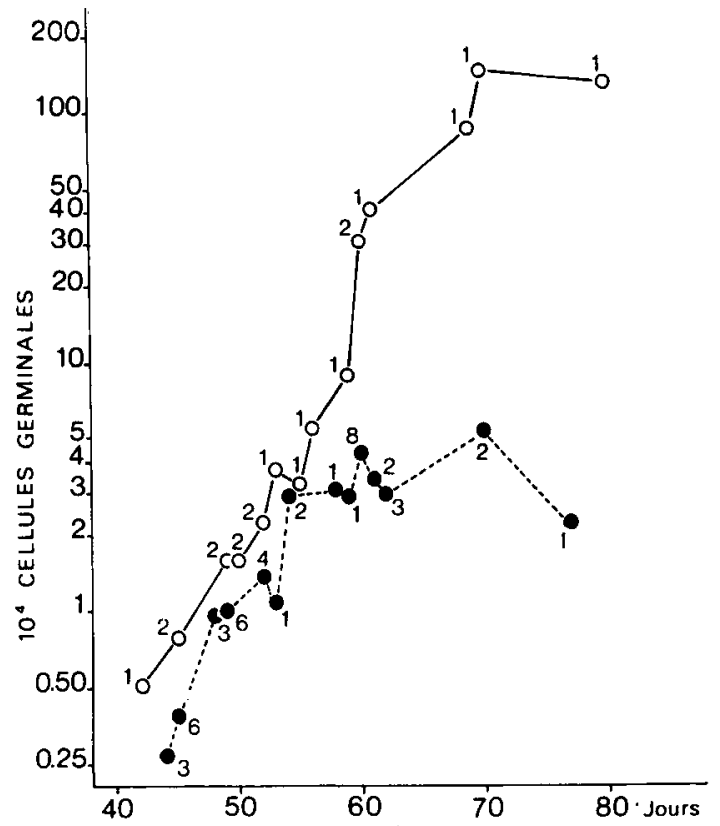

Frg. 2. - Nombre de cellules germinales par gonade, estimé selon la méthode de Chalkley, dans les gonades de freemartins (points noirs) et de femelles témoins (cercles)

Le nombre de fœetus étudiés est indiqué (ordonnées logarithmiques) 
germinales, surtout dans la zone périphérique. Leur nombre est multiplié par près de 20 entre 50 et 60 jours (fig. 2 ), alors que le volume gonadique n'est multiplié que par 3 (fig. I). Il s'ensuit que les cellules germinales restent en groupes, ou en " nids ", dans la zone périphérique, sous l'épithélium de surface. Le même processus s'intensifie jusqu'à 70 jours (entre 50 et 70 jours le nombre des cellules germinales est multiplié par go et celui de la gonade par 6,5 ). Ces accumulations de cellules germinales dans la région périphérique de la gonade ont été interprétées autrefois comme traduisant la poussée de cordons sexuels à partir du prétendu "épithélium germinatif " qui leur aurait donné naissance.

A partir de 56 jours, on voit des cordons de cellules très éosinophiles (intensément colorées aussi par le violet de crésyl) entre le blastème central et les nids ovigères en formation; 1'aspect est variable selon les animaux. Ces "cordons sombres ", qui se voient plus nettement aux stades ultérieurs (fig. 3 et 4 ), jouent peut-être un rôle dans la folliculogenèse. Ce sont probablement ces formations que certains anciens auteurs, qui n'avaient pas étudié un nombre de stades suffisant, ont pris pour des " cordons sexuels primaires " en régression.

Chez les freemartins deux faits surviennent, qui sont probablement liés :

I $^{\circ}$ le volume de la gonade cesse d'augmenter entre 49 et 52 jours, et en moyenne reste constant jusqu'à 62 jours (fig. I) (cf. Jos' et al., I972) et probablement jusqu'à 90 jours (fig. 7). Cet arrêt de croissance des gonades touche surtout les zones périphériques, autour du blastème central. Il est concomitant du début de la régression de la partie supérieure des canaux de Müller et il y a une bonne corrélation entre les degrés d'inhibition de l'ébauche gonadique et celle des canaux de Müller (Jost et al., I972).

$2^{\circ}$ Le nombre des cellules germinales augmente jusque vers 54 jours dans la gonade des freemartins, mais ensuite il ne suit pas l'accroissement énorme qui a lieu chez les femelles témoins à partir de ce moment (fig. 2). Il semble que dans certains cas l'arrêt de croissance de la gonade précède l'arrêt de la multiplication des cellules germinales, mais les deux phénomènes sont presque simultanés.

\section{0 à 90 jours}

Chez les foetus mâles cette période correspond surtout à l'achèvement des connexions uro-génitales : différenciation du rete testis, des tubes droits (entre rete et cordons séminifères) et des connexions entre rete et canal de Wolff. Un épididyme est reconnaissable après 70 jours, sous la forme d'ondulations du canal de Wolff.

Chez les femelles, la multiplication des cellules germinales se ralentit après 70 jours, et les premières prophases méiotiques apparaissent après 75 jours. Le rete ovarii est formé de tubes pourvus d'une lumière après 70 jours, comme celui des mâles, mais son volume augmente beaucoup moins.

Chez les freemartins, la gonade reste très petite ; son volume moyen ne change guère entre 50 et 90 jours (fig. 5 et 7 ), mais il y a de grandes variations individuelles. Le rete est bien développé et il est entouré par un blastème de cellules dont certaines ont un abondant cytoplasme. Les cellules germinales sont relativement abondantes dans le rete et dans le blastème. En surface, l'aspect est variable : des îlots à cellules germinales, surmontés par un épithélium encore cylinđrique, peuvent être relative- 

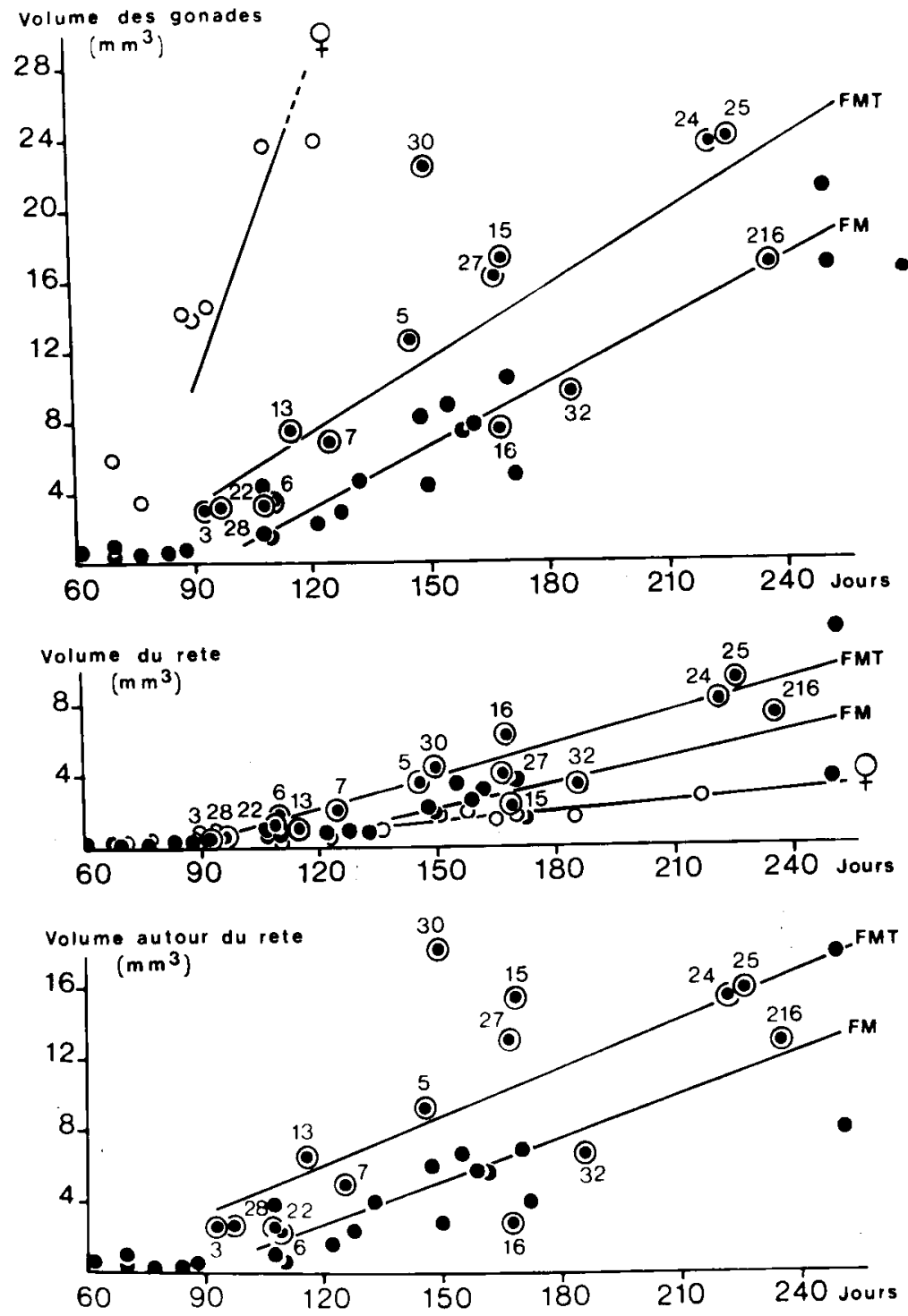

Fig. 7

En haut: volume moyen d'une gonade en fonction de l'âge $(O+=$ femelle témoin. $F M=$ freemartin dont la gonade ne contient pas de "cordon séminifère". FMT = freemartin dont la gonade contient de tels cordons ; le numéro de ces fœtus est indiqué dans les 3 graphiques).

La droite de régression des femelles témoins a été calculée pour I I fœetus de 9r à 2I 7 jours; celle des freemartins FM pour 15 fœetus de 108 à 251 jours; celle des freemartins FMT pour Is fœtus de 93 à 236 jours.

A $u$ milieu : volume moyen du rete des mêmes gonades : ce volume comprend l'ensemble du rete intraet extragonadique.

En bas: volume des moyen tissus entourant le rete des mêmes gonades.

Pour les trois figures : $\mathrm{O}=$ femelle témoin ; $\bullet$ freemartin dont la gonade ne contient pas de " cordon séminifère "; freemartin ayant de tels cordons : point noir cerclé de blanc. 
ment nombreux (cas d'un freemartin de 70 jours), ou présents seulement dans une ou deux régions localisées de la gonade ; ailleurs la surface ressemble alors davantage à une albuginée testiculaire (fig. 5). Dans un freemartin de 77 jours, quelques cellules germinales étaient en prophase méiotique dans une région limitée de la périphérie (fig. 5 et 6); mais le fait semble rare.

\section{Fatus de plus de 90 jours}

Le đébut du quatrième mois correspond à des événements morphogénétiques importants chez les foetus femelles et chez certains freemartins.

Chez la femelle va commencer la différenciation ovarienne, c'est-à-dire la formation de follicules séparés par un stroma ovarien. On trouve un très petit nombre de follicules, dans la profondeur de la zone ovigère, à partir du stade de Ioo à Iro jours. Leur nombre va en augmentant pendant les semaines suivantes. Les " cordons sombres " décrits plus haut sont venus en contact avec la zone où se forment les follicules (fig. 4) mais, pour le moment, nous ne pouvons préciser leur rôle dans l'apparition des cellules folliculaires. La période d'organogenèse ovarienne s'accompagne d'une croissance rapide de l'ovaire (fig. 7), après 1'arrêt de la multiplication des cellules germinales.

Après go jours, la croissance des gonades des freemartins reprend (fig. 5 et 7 ). On peut distinguer trois parties dans ces gonades :

${ }^{\circ}$ en position centrale ou plus souvent excentrique, un rete qui paraît très volumineux étant donné la petite taille des gonades; après I2o jours le rete peut atteindre un volume double ou triple de celui trouvé dans un ovaire normal (fig. 7). Le rete des mâles est encore beaucoup plus volumineux;

$2^{\circ}$ en périphérie, une albuginée très semblable à celle d'un testicule; les zones où subsiste un épithélium de type ovarien surmontant des nids de cellules germinales sont exceptionnelles ;

$3^{\circ}$ autour $d u$ rete, un blastème cellulaire plus ou moins abondant (fig. 7). Parfois ce blastème est très réduit (fig. 5,84 j) et l'on peut supposer que ces gonades resteront rudimentaires et indifférenciées, comme on le voit chez certains freemartins adultes (LILIIE, I9I7 ; WILLIER, I92I). Dans d'autres gonades de IO0 à 250 jours, ce blastème bien que plus abondant, ne renferme pas de structure reconnaissable. Enfin, dans la moitié environ des freemartins étudiés entre 90 et 250 jours, ce blastème contient des cordons qui ressemblent soit aux cordons séminifères déjà bien différenciés des mâles de roo jours, soit aux cordons encore en formation, visibles dans les testicules des mâles de 42 à 45 jours (fig. 6). On peut faire les remarques suivantes au sujet de ces structures :

a) dans l'ensemble, ces cordons existent surtout dans les gonades ayant un blastème abondant autour du rete (fig. 7);

b) le freemartin le plus jeune ayant de tels cordons avait un âge estimé de 93 jours ; le freemartin de 97 jours est l'un de ceux qui possédait les cordons séminifères les mieux différenciés et les plus nombreux. La répartition des cordons séminifères bien différenciés et des cordons en formation a été la suivante :

- Chez 3 freemartins $(97 \mathrm{j} ; 150 \mathrm{j} ; 236 \mathrm{j}$ ), les gonades renferment un grand 
nombre de cordons séminifères bien différenciés, en relation avec le rete (fig. 5 ). Dans les deux premiers, il y a des îlots de cellules ressemblant à des cellules interstitielles testiculaires.

- Chez 4 freemartins ( $109 \mathrm{j} ; \mathrm{I} 68 \mathrm{j} ; \mathrm{I} 69 \mathrm{j} ; 226 \mathrm{j}$ ), il existe un petit nombre de cordons séminifères bien différenciés; ces cordons ne sont pas tous en relation avec le rete.

- Chez 6 freemartins (II5 j; I25 j ; I46 j; I67 j ; I86 j ; $222 \mathrm{j}$ ), coexistent des cordons séminifères bien différenciés et des cordons irréguliers de diamètre plus faible (fig. 6), que nous considérons comme des cordons séminifères en formation. Cette interprétation est appuyée par les cas où les deux sortes de structures sont en continuité. Ces différenciations s'observent soit isolées parmi les cellules du blastème, soit au contact du rete. Io8 j).

- Enfin chez deux freemartins n'existent que des cordons en formation (93 $j$;

Si ce que nous avons appelé " cordons en formation " représente bien un stade initial dans la formation des cordons séminifères, il semble que ceux-ci puissent se différencier dans la gonade des freemartins pendant une période prolongée; on trouve en effet des cordons en formation chez des freemartins d'âge variant entre 93 et 222 jours. Il se peut qu'il en apparaisse encore ultérieurement ;

c) les cordons séminifères des freemartins les plus jeunes renferment un nombre appréciable de cellules germinales. Le nombre de ces cellules devient plus faible après $I_{50}$ jours et leur présence est rare après 200 jours. Il semble donc que certains de ces cordons puissent se former par la différenciation et l'association des cellules claires qui les constituent, avec une participation très faible ou même nulle des cellules germinales. Dans les testicules des mâles normaux de 42 ou 43 jours, on voit aussi s'ébaucher certains futurs tubes séminifères par l'association de cellules claires, qui sans doute deviendront les cellules de Sertoli, et seulement d'un très petit nombre de cellules germinales ;

d) jusqu'à l'âge de 62 jours, l'appareil génital des freemartins ne montre pratiquement pas de signe de masculinisation (Jost et al., I972). Nous avons maintenant étudié l'appareil génital de I3 freemartins âgés de 70 à I25 jours, et fait les observations suivantes :

- Chez les 4 freemartins les plus jeunes $(70,70,77$ et 88 jours) la masculinisation, si elle existe, est encore peu marquée. Les vésicules séminales ne sont pas ramifiées comme chez les mâles, et leur longueur n'est nettement augmentée que chez le plus âgé.

- Chez 3 freemartins plus âgés, dont les gonades ne contenaient pas de " cordons séminifères " (I08, ro8 et I Io jours), les vésicules séminales sont longues et ramifiées ; 1'épididyme est indiqué dans deux cas.

- 3 freemartins dont les gonades contenaient quelques cordons en formation (93, Io8 jours) ou seulement 3 cordons séminifères différenciés (Iog j), ressemblent aux précédents mais montrent en plus un ou deux bourgeons prostatiques.

- Chez les 3 freemartins dont la gonade était la plus transformée dans le sens testiculaire (97, II5 et I25 j), les signes de masculinisation sont plus prononcés : les vésicules séminales ramifiées sont presque deux fois plus longues que dans les cas 
précédents, et, chez les detux freemartins dont la gonade contenait des îlots de cellules interstitielles, l'épididyme et la prostate étaient très développés.

Ces observations confirment nos résultats antérieurs, en montrant que la masculinisation de l'appareil génital des freemartins ne survient que tardivement et qu'elle ne porte que sur les structures qui sont encore capables de répondre aux androgènes après 60 jours (JOST et al., I973). Elles montrent en plus que le degré de masculinisation de l'appareil génital est le plus fort chez les freemartins dont les gonades sont elles-mêmes les plus masculinisées. Ce parallélisme pourrait s'expliquer par une action masculinisante particulièrement puissante après 90 jours qui s'exercerait soit à la fois sur les gonades et sur l'appareil génital, soit seulement sur les gonades qui, à leur tour, stimuleraient certaines parties de 1'appareil génital. On notera cependant que les vésicules séminales et l'épididyme peuvent montrer une stimulation androgénique même en 1'absence de masculinisation reconnaissable de la gonade.

\section{CONCLUSIONS ET INTERPRÉTATION}

L'histoire des freemartins comporte quatre périodes essentielles :

- pendant la première semaine suivant la différenciation testiculaire des mâles, les freemartins restent très semblables aux femelles normales ;

- entre 48 et 60 jours environ, la croissance de la gonade des freemartins et la multiplication des cellules germinales s'arrêtent; la partie antérieure des canaux de Müller régresse, mais il n'y a aucun signe de masculinisation;

- entre 60 et 90 jours, la gonade des freemartins est toujours très involuée, mais une légère stimulation des vésicules séminales ou de l'épididyme s'observe parfois ;

- après go jours, la croissance des gonades des freemartins reprend, en parallèle avec une forte croissance des gonades des fœetus femelles ; dans environ la moitié des cas apparaissent des formations ressemblant à des cordons séminifères testiculaires, vers l'époque où chez les femelles normales se différencient les follicules ovariens.

Les neuf freemartins de plus de go jours dont nous avons étudié le tractus génital montrent tous des signes de masculinisation au niveau des vésicules séminales; certains, aussi au niveau de l'épididyme. Ceux qui ont la gonade la plus transformée dans le sens testiculaire ont aussi les vésicules séminales et les épididymes les plus stimulés et ont développé des bourgeons prostatiques. De plus, les gonades qui contiennent des cordons de type testiculaire sont généralement celles dont le rete est le mieux développé et dont le volume est le plus gros (fig. 7). Il n'est pas impossible qu'il y ait une relation causale entre l'état du rete et le développement des cordons testiculaires. De toute manière, ces observations peuvent prêter à diverses interprétations sur les causes du freemartinisme, mais aucune ne peut actuellement être considérée comme démontrée et il est inutile de les discuter aujourd'hui (cf. JosT et al., I972, I973; VIGIER et al., I972). La connaissance, maintenant acquise, de la 
chronologie et des modalités morphologiques des phénomènes d'organogenèse particuliers à la gonade des freemartins, devrait permettre une étude ultérieure des facteurs en cause.

\section{REMERCIEMENTS}

Le présent travail a été rendu possible par un contrat de la D. G. R. S. T. et grâce à la collaboration constante des Stations de Physiologie de la Reproduction de l'I. N. R. A. de Jouy en Josas et de Nouzilly, en particulier du professeur Ch. Thrbault, de MM. R. Ortavant, P. MauLéon, F. du Mesnil du Buisson, Y. de Fontaubert, F. de La Chevalerie, J. Pont, P. Chesne, R. Scandolo et de Mme M. Gtrard, que nous remercions cordialement.

\section{SUMMARY}

\section{THE DEVELOPMENT OF THE GONAD OF FREEMARTINS}

The differentiation of the gonads has been studied in 82 fetal freemartins preserved at ages between 40 and 250 days after insemination. Twin or multiple pregnancies with fetuses of opposite sexe were either induced with gonadotrophins or obtained at the slaughter house.

The history of the fetal freemartin gonads can be divided in four periods.

During the week following the onset of testicular differentiation in male fetuses (days 4048 ), the gonads of the presumptive freemartins remain similar to the undifferentiated gonads of control female fetuses.

Between days 48 and 60 , gonadal growth and multiplication of germ cells stop. Simultaneously the upper part of the Mullerian ducts regresses, but no masculinization of the gonads or of the genital tract can be recognized.

Between days 60 and 90 , the freemartin gonad remains stunted and the last peripherical nests of germ cells disappear. Some slight signs of androgenic stimulation can be seen at the level of the seminal vesicles and less frequently of the epididymis.

After 90 days, when ovarian follicles start differentiating in normal females, growth of the freemartin gonad resumes. In approximately half the cases, the gonads contain cellular cords resembling seminiferous cords at varying degrees of differentiation.

These cords are either isolated in the gonadal blastema or connected with the rete. In all these cases the rete is enlarged. In a few cases, clusters of interstitial cells of the testicular type were present. In the three freemartins in which the gonads were the most transformed in the testicular direction, the rete was the largest and the genital tract the most masculinized ; in particular a prostate had developed.

\section{RÉFÉREINCES BIBLIOGRAPHIQUES}

VAN BeEk W. F., r924. Die Entwicklung des Eierstockes beim Rinde. Zeitsch. Anat. Entwicklungsgeschichte, 71, 458-558.

Bissonnette T. H., I924. The development of the reproductive ducts and canals in the freemartin with comparison of the normal. Amer. J. Anat., 33, 267-345.

Bissonnette T. H., 1928. Notes on multiple pregnancies in cattle, with special reference to three cases of prenatal triplets and the freemartins involved. Amer. $J$. Anat., 42, 29-73.

CHALKLEY H. W., I943. Method for the quantitative morphologic analysis of tissues. J. Nat. Cancer Instit., 4, 47-53.

Chapis C. L., I9I7. A microscopic study of the reproductive system of fetal freemartins. J. Exp. Zool., $23,453-482$.

ErICKSON B. H., I966. Development and radio-response of the prenatal bovine ovary. J. Reprod. Fert., 11, 97-105. 
Fischel A., 1930. Über die Entwicklung der Keimdrüsen des Menschen. Z, Anat. Entwickl. Gesch., 92, 34-72.

Gropp A., OHNo S., I966. The presence of a common embryonic blastema for ovarian and testicular parenchymal (follicular, interstitial and tubular) cells in cattle, Bos Taurus. Zschr. Zellforsch., 74, 505-528.

HAY D., 1950. Étude d'un freemartin spontané chez les ruminants. Arch. Anat. Histol. Embryol. (Strasbourg), 33, 53-80.

Jost A., I970. Hormonal factors in the sex differentiation of the Mammalian fotus. In " Discussion on Determination of Sex ". Phil. Trans. Roy. Soc. Lond., B., 259, 1 19-130.

Jost A., Vigier B., Prepin J., I972. Freemartins in cattle : the first steps of sexual organogenesis. J. Reprod. Fert., 29, 349-379.

Jost A., Vigier B., Prepin J., Perchellet J. P., r973. Studies on Sex Differentiation in Mammals. Rec. Progr. Horm. Res. (sous presse).

LILliE F., Igr7. The freemartin, a study of the action of sex hormones in fetal life of cattle. J.exp. Zool., 28, 37I-45I.

Lillie F. R., r923. Supplementary notes on twins in cattle. Biol. Bull., 44, 47-48.

Maulton P., Mariana J.-C., Benoit M., Solari A., Chupin D., 197o. Influence de différentes doses de PMSG et HCG injectées en phase folliculaire du cycle cestrien, sur le nombre et le rendement d'ovulations de vaches de race Française Frisonne pie noire. Ann. Biol. anim. Biochim. Biophys., 10, suppl. I, 3I-46.

Ohno S., Trujillo J., Stenius C., Christian L. C., Teplitz R., I962. Possible germ cell chimeras among newborn dizygotic twin calves (Bos Taurus). Cytogenetics, 1, 258-265.

OHNo S., I969. The problem of the bovine freemartin. J. Reprod. Fert. Suppl., 7, 53-6r.

Prenant A., I889. Contribution à l'histogenèse du tube séminifère. Internat. Mschr. Anat. Physiol., 6, I-30.

Rajakoski E., Hafez E. S. E., r963. Derivatives of Cortical Cords in Adult Freemartin Gonads of Bovine Quintuplets. Anat. Rec., 147, 457-467.

Short R. V., Smith J., Mann T., Evans E. P., Hallett J., Fryer A., Hamerton J. L., I969. Cytogenetic and endocrine studies of a freemartin heifer and its bull co-twin. Cytogenetics, 8, 369-388.

Vigier B., Prepin J., Jost A., I972. Absence de corrélation entre le chimérisme XX/XY dans le foie et les premiers signes de freemartinisme chez le fetus de Veau. Cytogenetics, 11, 8r-ror.

Willier B. H., I921. Structures and homologies of freemartin gonads. J. exp. Zool., 33, 63-127.

\section{PLANCHE I}

FIG. 3

Coupe de l'ébauche ovarienne d'un fotus femelle témoin de 63 jours, montrant les " nids ovigères " en périphérie et 1'organisation de cordons dans le blastème central.

CM : Canal de Müller ; CW : Canal de Wolff.

Même grossissement que figure $6(\times 36)$.

FIG. 4

Détail du contact entre " cordons sombres " et les nids ovigères (en haut) dans l'ébauche ovarienne d'un fotus femelle témoin de 95 jours $(\times 270)$. 

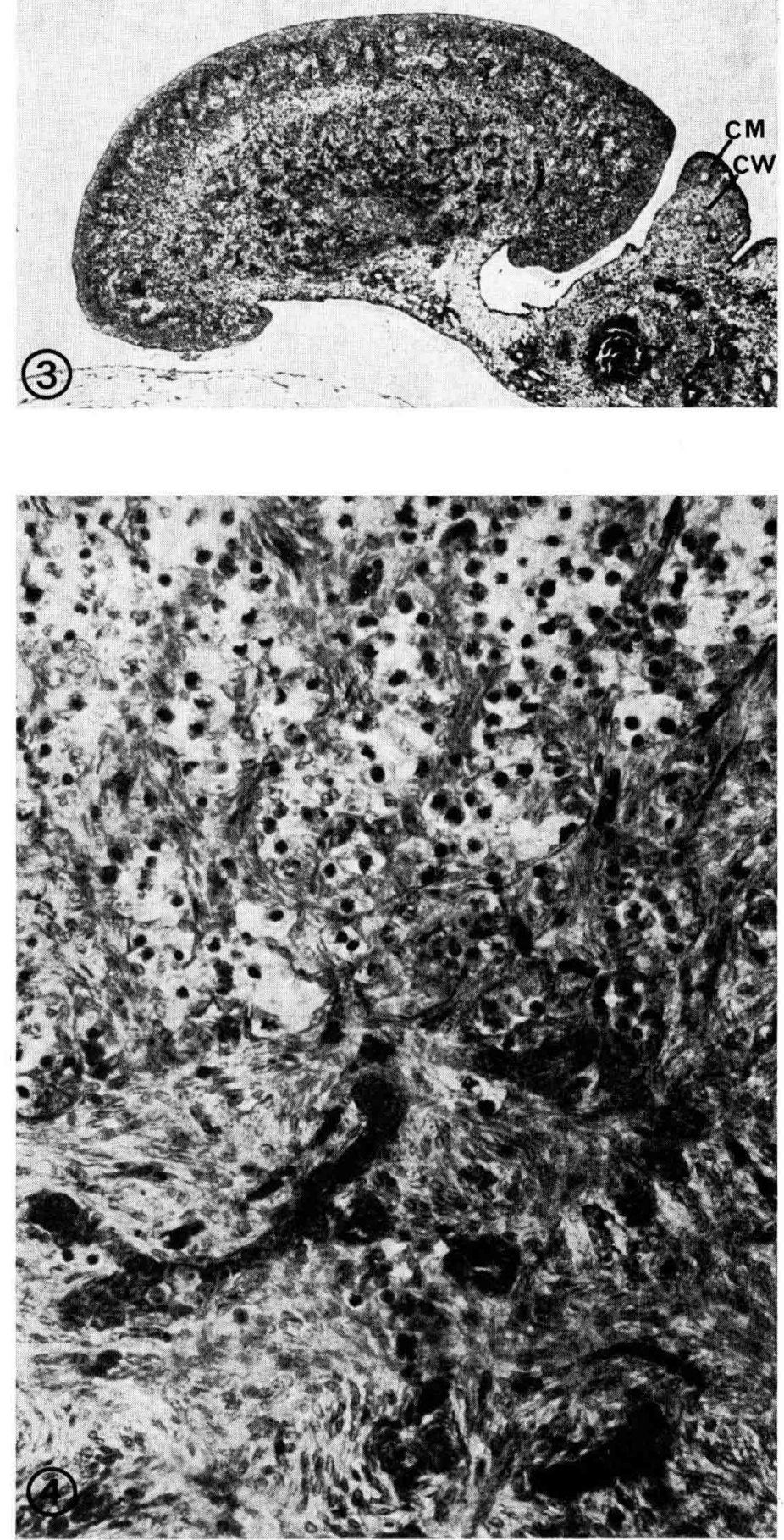

A. JOST, B. VIGIER, J. PRÉPIN et J. P. PERCHELLET 


\section{PLANCHE II}

FIG. 5

Coupes à travers les gonades de cinq freemartins dont l'âge en jours est indiqué. Le volume de ces gonades est le suivant : 77 jours : $0,47 \mathrm{~mm}^{3} ; 84$ jours : $0,67 \mathrm{~mm}^{3} ; 88$ jours : $0,87 \mathrm{~mm}^{3}$; 97 jours : $3,20 \mathrm{~mm}^{3} ; 150$ jours : $22,30 \mathrm{~mm}^{3}$. Les gonades de 97 et de 150 jours, contenant de nombreux cordons séminifères, portent respectivement les numéros 28 et 30 dans la figure 7. Dans les fotus de plus de 77 jours on reconnait le rete $(R)$. Un détail de la gonade, indiqué par une flèche, se trouve dans la figure 6. Grossissement : $\times 36$. 
PLANCHE II
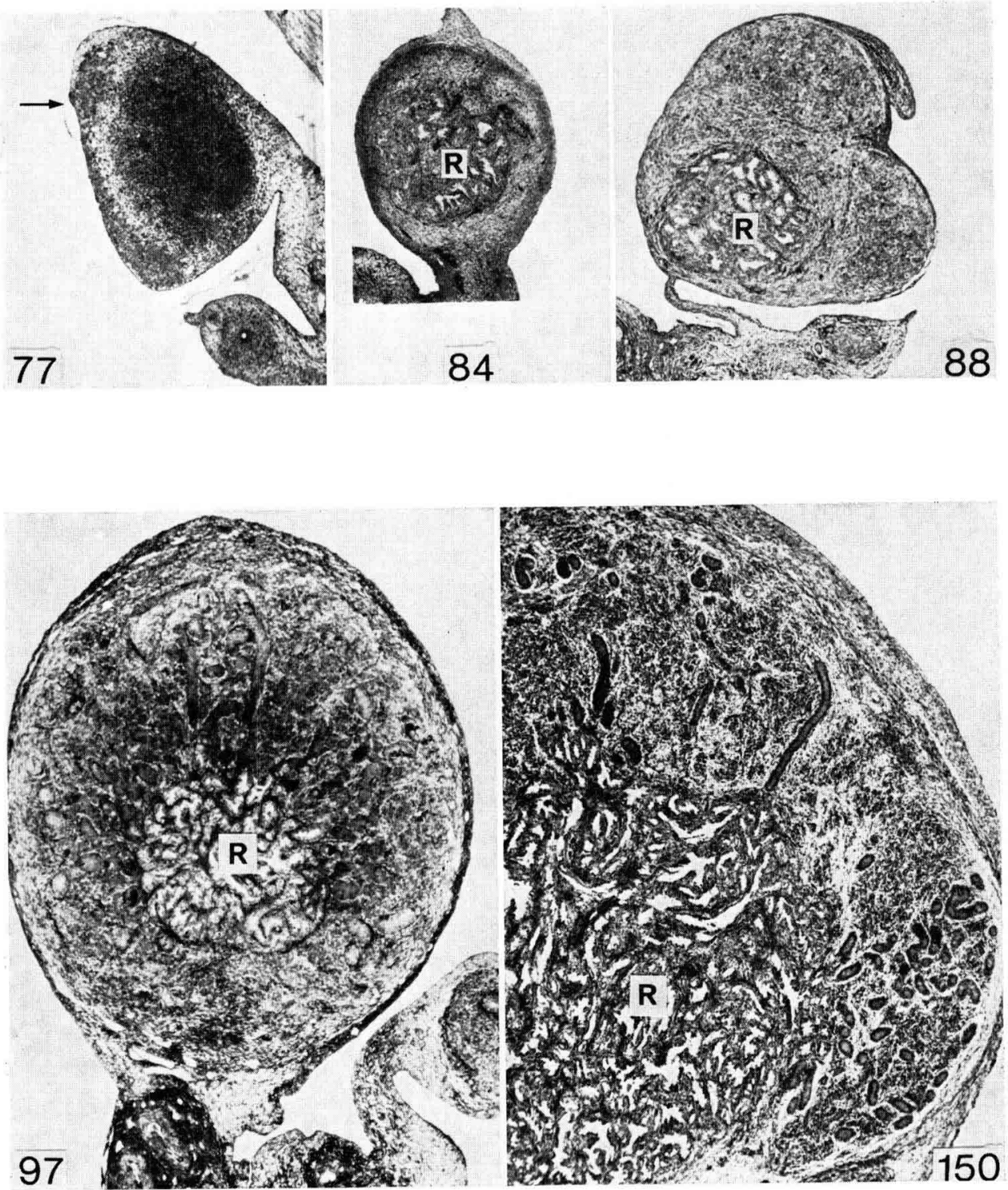

A. JOST, B. VIGIER, J. PRÉPIN et J. P. PERCHELLET 


\title{
PLANCHE III
}

\author{
FIG. 6
}

Détail des gonades de 4 freemartins dont l'âge en jours est indiqué, et montrant : 1) un nid à ovocytes dans une gonade de 77 jours (cf. Fig. 5) ; 2 ) un cordon séminifère bien organisê (97 jours) et sa jonction avec le rete (R); 3 ) des "cordons en formation" (à 186 et 222 jours), à des niveaux choisis de manière à montrer des cellules germinales. Grossissement : $\times 450$. 


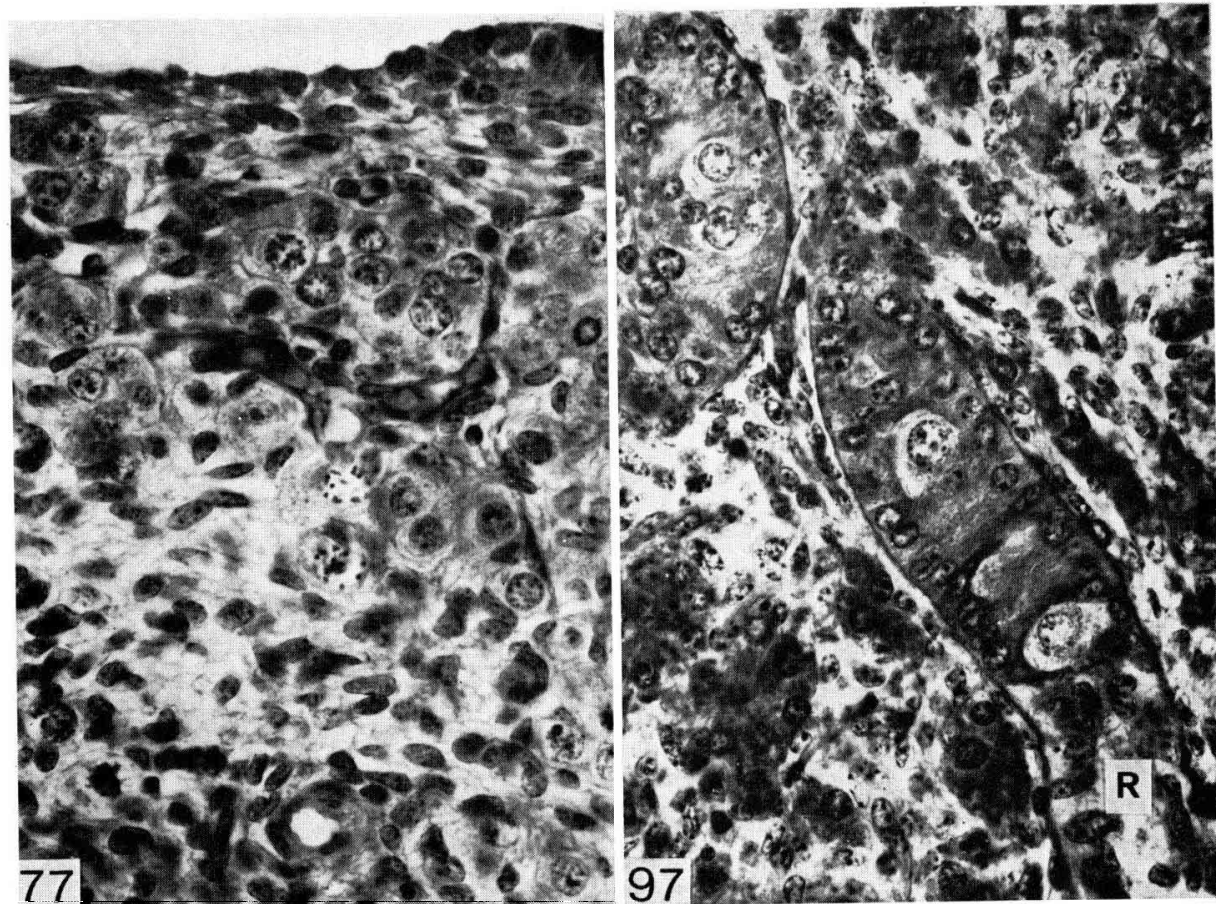

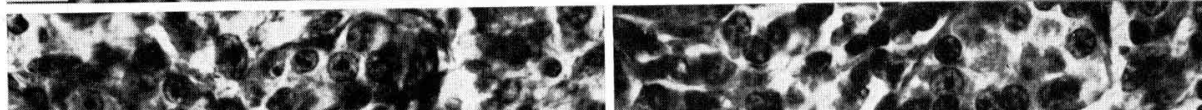

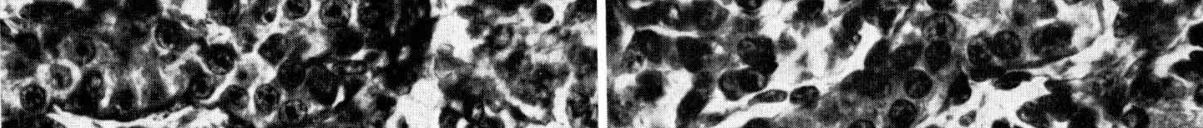

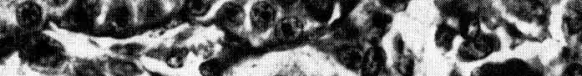

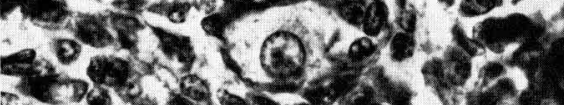
2.

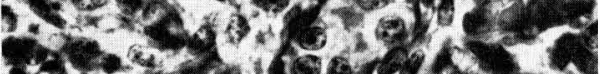

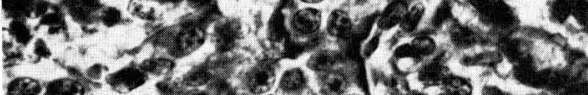

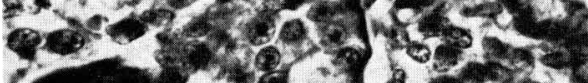

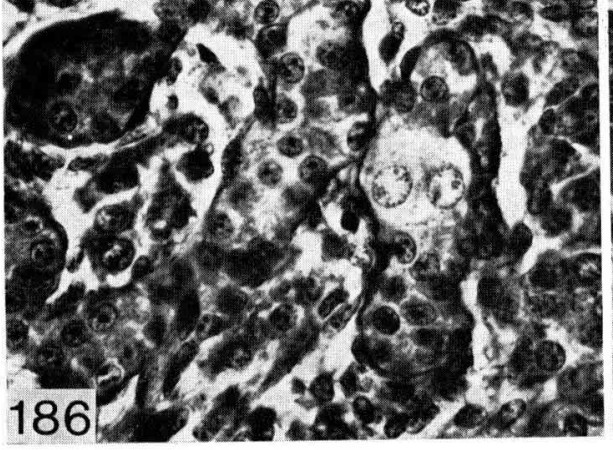

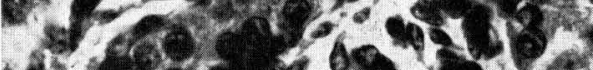

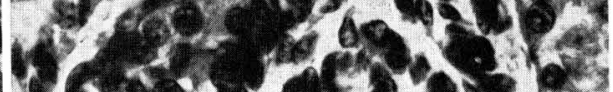

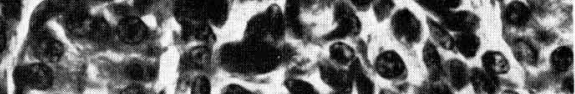

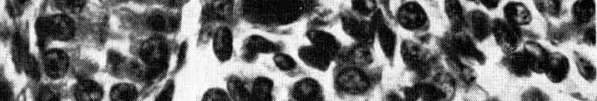

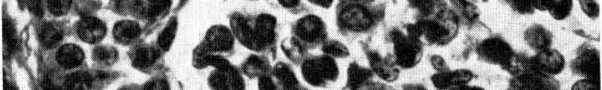

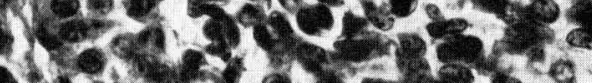

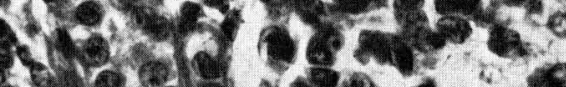
$3 e^{3}$ a a

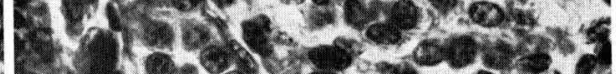

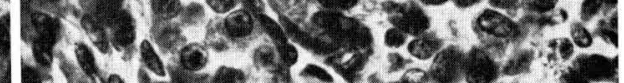

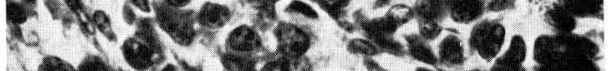

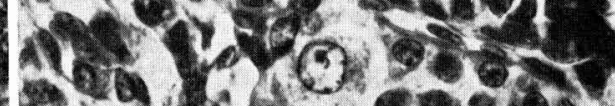
a

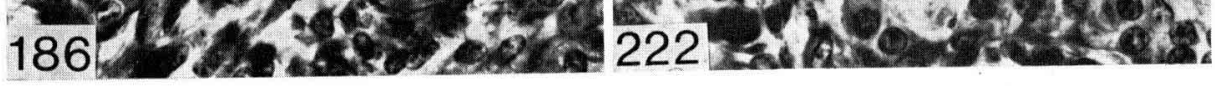

A. JOST, B. VIGIER, J. PRÉPIN et J. P. PERCHELLET 